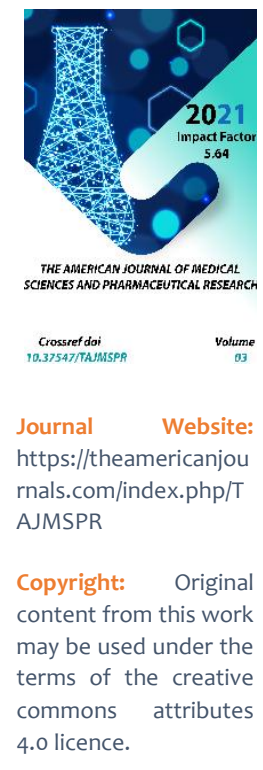

\section{Modern Tactics Of Treatment Of Patients With Epilepsy}

Yakutkhon Nabievna Madjidova

Doctor Of Medical Sciences, Professor, Head Of The Department Of Neurology, Tashkent Pediatric Medical Institute, Uzbekistan

Shokirjon Bakhodirovich Mamatkurbonov

Neurologist Of The Surkhandarya Branch Of The Republican Scientific Center Of Emergency Care, Assistant Of The Department Of Neurology, Termez Branch Of The Tashkent Medical Academy, Uzbekistan

\title{
ABSTRACT
}

This article explores modern methods of treating epilepsy. The problem of epilepsy is one of the most urgent in modern neurology and psychiatry. According to the European Commission on Epilepsy, about 50 million people in the world suffer from epilepsy. Epilepsy has attracted the attention of various specialists for many centuries, due to the spread of this disease in the population, the complexity of the etiology and pathogenesis, the polymorphism of clinical manifestations, the severity of the course, potentially severe clinical and social consequences, insufficiently effective treatment. According to WHO, the lack of adequate information on the epidemiological characteristics of epilepsy in many countries causes significant deficiencies in the organization of medical care. Epilepsy is one of the most common and severe diseases of the nervous system. The risk of developing epilepsy varies greatly with age. The peak incidence of epilepsy occurs in childhood and adolescence. After the second decade of life, the risk of epilepsy is significantly reduced. However, in some cases, epileptic seizures persist in patients until old age, and can also occur for the first time in old and senile age.

\section{KEYWORDS}

Lack of sleep, physical and mental stress, hyperthermia, epilepsy, nervous system, medical care.

\section{INTRODUCTION}

Epilepsy treatment has two directions - medical and surgical.

Medication consists in prescribing a complex of anticonvulsants that affect a specific area of the brain (depending on the localization of the pathological focus). The main task of such therapy is to stop or significantly reduce the number of attacks. Medicines are prescribed 
individually, depending on the type of epilepsy, the patient's age and other physiological characteristics.

The goal of treatment is to stop epileptic seizures with minimal side effects and to manage the patient in such a way that his life is as fulfilling and productive as possible.

Modern tactics of treating patients with epilepsy include the following:

- Identifying those causes of seizures that are treatable (tumor, aneurysm, etc.);

- Exclusion of factors provoking seizures (lack of sleep, physical and mental stress, hyperthermia);

- Correct diagnosis of the type of epileptic seizures and epilepsy;

- Prescribing adequate drug therapy (inpatient or outpatient);

- Attention to education, employment, rest of patients, social problems of a patient with epilepsy.

Treatment principles for epilepsy:

- Compliance of the drug with the type of seizures and epilepsy (each drug has a certain selectivity in relation to one or another type of seizure and epilepsy);

- Use of monotherapy if possible (use of one antiepileptic drug.

Conservative treatment: Treatment should begin with the appointment of a small dose of an antiepileptic drug recommended for this type of seizure and the form of epilepsy. The dose is increased if there are no side effects and the seizures remain in general. In recent years, many new antiepileptic drugs have appeared which are characterized by higher efficacy and better tolerance.

Indications for the use of anticovulsants:
1. Treatment of patients with one paroxysm (presumptive diagnosis “epilepsy”) with a combination of the following symptoms:

- The patient has a history of febrile seizures;

- The presence of a family history of epilepsy;

- impaired mental function;

- Focal neurological symptoms;

- When detecting "epileptiform” EEG patterns.

2. Treatment of patients with a wellestablished diagnosis of epilepsy (the presence of repeated non-provoked seizures, exclusion of seizures of nonepileptic genesis).

3. Treatment of patients with one or more provoked attacks due to an acute illness or condition (encephalitis, withdrawal syndrome, use of convulsive drugs) - while the treatment of the underlying disease continues.

4. Preventive treatment of patients with diseases or conditions that have a high likelihood of seizures or epilepsy (cranial injury, neurosurgical intervention, stroke, encephalitis) - start anticonvulsant therapy only when epileptic seizures occur.

\section{THE MAIN FINDINGS AND RESULTS}

\section{Prevention of epilepsy}

Preventive measures:

1. Preventing head injury is the most effective way to prevent post-traumatic epilepsy.

2. Adequate perinatal care helps to reduce the number of new cases of epilepsy due to birth trauma.

3. The use of drugs and other methods of lowering body temperature in children with fever may reduce the likelihood of febrile seizures. 
4. Central nervous system infections are a common cause of epilepsy in tropical regions, where many developing countries are located.

5. The destruction of parasites in these conditions and educational work regarding the prevention of infections are effective ways to reduce the burden of epilepsy worldwide, for example, epilepsy caused by neurocysticercosis.
For the prevention of epilepsy, the fairer sex with epileptiform manifestations should take mild diuretics in 10-day courses during the menstrual cycle ( 3 days before the onset of menstruation, 4-5 days during them and another 2-3 days after the end of menstruation).

\section{Clinical signs of epilepsy}

Fig. 1 Types of Epileptic Seizures

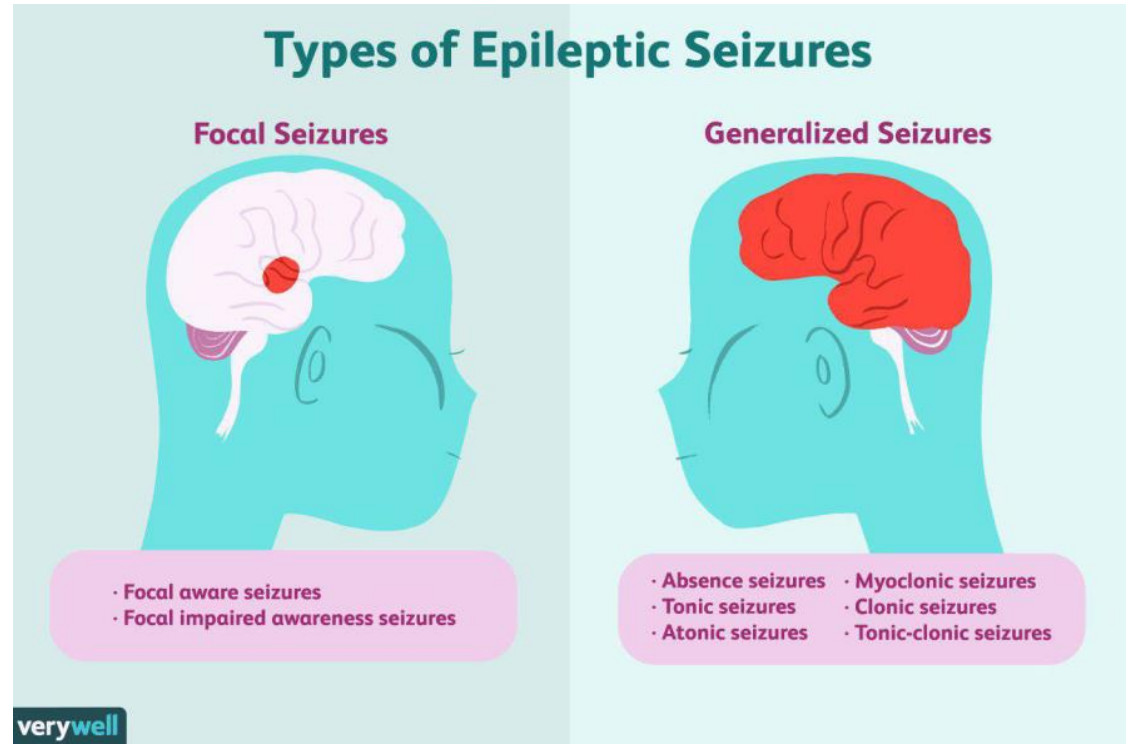

In the clinical picture of epilepsy, a seizure or seizure period and an interictal period are distinguished. It should be emphasized that in the interictal period, neurological symptoms may be absent or determined by the disease causing epilepsy (traumatic brain injury, stroke, etc.)

The most common symptom of epilepsy is a major seizure. It usually begins suddenly, and its onset is not associated with any external factors. Less commonly, distant precursors of a seizure can be identified. In these cases, 1-2 days before it, there is a feeling of poor health, headache, sleep disturbance, appetite disturbance, and increased irritability. In most patients, the seizure begins with the appearance of an aura, which is stereotyped in the same patient.

Depending on the irritation of the brain area from which the epileptic discharge begins, several main types of aura are distinguished: autonomic, motor, mental, speech and sensory (fig 2). 


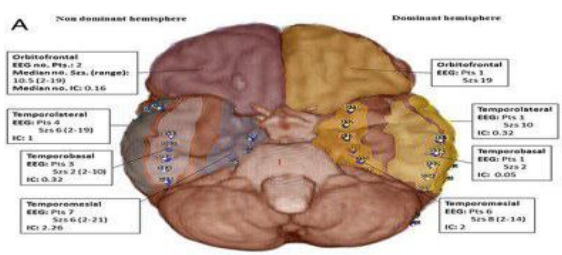

c

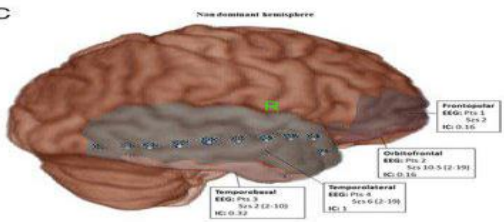

E

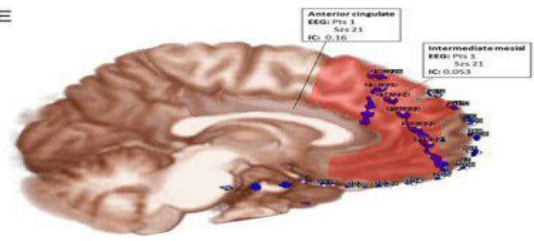

B
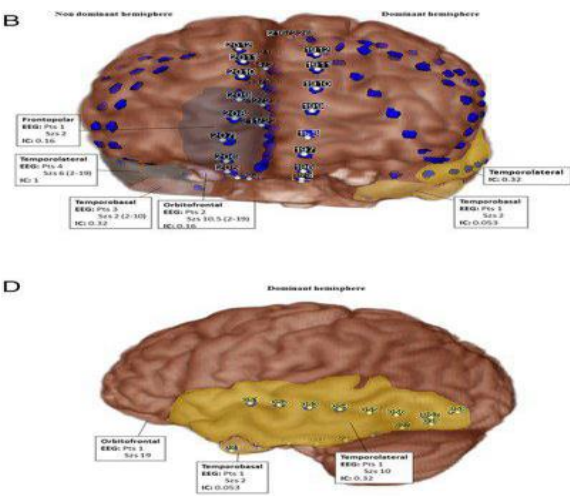

After the passage of the aura, which lasts for several seconds, the patient loses consciousness and falls as if knocked down. The fall is accompanied by a kind of loud cry caused by spasm of the glottis and convulsive contraction of the chest muscles.

Convulsions immediately appear, at first tonic: the trunk and limbs are stretched in a state of tension, the head is thrown back and sometimes turns to the side, breathing is delayed, the veins in the neck swell, the face becomes deathly pale, with gradually increasing cyanosis, the jaws are convulsively clenched.

The tonic phase of the seizure lasts $15-20 \mathrm{~s}$. Then clonic convulsions appear in the form of jerky contractions of the muscles of the limbs, neck and trunk.

During the clonic phase of a seizure lasting up to 2-3 minutes, breathing is often hoarse, noisy due to accumulation of saliva and retraction of the tongue, cyanosis slowly disappears, foam comes out of the mouth, often stained with blood due to biting the tongue or cheek.
The frequency of clonic seizures gradually decreases, and at the end of them, general muscle relaxation occurs. During this period, the patient does not react even to the strongest stimuli, the pupils are dilated, their reaction to light is absent, tendon and protective reflexes are not triggered, involuntary urination is often noted. Consciousness remains soporous and only after a few minutes gradually clears up. Often, coming out of a soporous state, the patient plunges into deep sleep. At the end of the seizure, they complain more about fatigue, lethargy, drowsiness, but they do not remember anything about the seizure itself.

Symptoms in partial seizures are determined by the syndrome of irritation of any area of the cortex of the diseased brain. Among the simple partial seizures, the following can be distinguished: with motor signs; with somatosensory or specific sensory symptoms (sounds, flashes of light or lightning); with vegetative symptoms or signs (peculiar sensations in the epigastrium, pallor, sweating, skin redness, piloerection, mydriasis); with 
mental symptoms. Complex seizures are characterized by varying degrees of impairment of consciousness. In this case, consciousness may not be completely lost, the patient partially understands what is happening around. Often complex partial seizures are caused by a focus in the temporal or frontal lobe and begin with an aura.

The sensory aura includes a variety of perceptual disorders. The visual aura that occurs when the occipital lobe is affected is usually manifested by the vision of bright sparks, shiny balls, ribbons, bright red coloring of surrounding objects (simple visual hallucinations) or in the form of images of some persons, individual parts of the body, figures (complex visual hallucinations) ... The sizes of objects change (macro- or micropsia). Sometimes the fields of vision fall out (hemianopsia), complete loss of vision is possible (amaurosis). With the olfactory aura (temporal lobe epilepsy), patients are haunted by a "bad" smell, often in combination with gustatory hallucinations (taste of blood, bitterness of metal, etc.).

\section{CONCLUSION}

Epilepsy is a chronic, non-infectious brain disorder that affects people of all ages. One single seizure does not mean epilepsy (worldwide, up to $10 \%$ of people have had one seizure during their lifetime). The definition of epilepsy applies to 2 or more unprovoked seizures. Epilepsy is one of the oldest recognizable conditions in the world, with written records dating back to 4000 BC. For centuries, epilepsy has been surrounded by fear, misunderstanding, discrimination and social stigma. In many countries, some forms of stigma continue to this day and can affect the quality of life of people with the disorder and their families.

According to WHO, the lack of adequate information on the epidemiological characteristics of epilepsy in many countries causes significant deficiencies in the organization of medical care. According to most authors, $60-80 \%$ of patients manage to achieve drug remission. Most of the developed antiepileptic drugs are approved for use in the Republic of Uzbekistan, that is, patients with epilepsy have the necessary conditions to achieve adequate remission.

\section{REFERENCES}

1. Agranovich O.V. On the issue of organizing specialized epileptological care for children, Medical Bulletin of the North Caucasus, 2015; (3) -pp 2630.

2. Bavdurny. A.A. Partial epilepsies: epidemiology, diagnosis, treatment, social aspects // Abstracts of the IIV Russian National Congress "Man and Medicine". Moscow: 2016. -pp. 65-71.

3. Volkov I.V. Experience of organizing antiepileptic care in Novosibirsk, Bulletin of Epileptology, 2017; 1 (02) pp. $15-7$

4. Voronkova K.V. Problems in the treatment of patients with epilepsy and their solution, Epilepsy, 2015; 17 (2), - 3 -p. 8.

5. Alekseevna, G. T., Nabiyevna, M. Y., \& Turgunovna, K. N. (2021). Efficiency of Microcurrent Reflexotherapy in Children with Autism Spectrum Disorders. Annals of the Romanian Society for Cell Biology, 4803-4813.

6. Milchakova. L.E. Quality of life in pharmacoeconomic studies of epilepsy 
The American Journal of Medical Sciences and Pharmaceutical Research

(ISSN - 2689-1026)

Published: September 30, 2021 | Pages: 96-101

IMPACT FACTOR

2021: 5.64

Doi: https://doi.org/10.37547/TAJMSPR/Volume03Issue09-16

II IV All-Russian Congress of

Pharmacoeconomics at the turn of the

third millennium. Conference

materials. Moscow: 2017. -pp. 125.

7. Kim, O., Madjidova, Y., Abidova, M., Hamidova, N., Abdullaeva, V., \& Sattarov, T. (2021). ClinicalNeurological and neurocognitive indicators against the background of neuroprotective therapy in vertebrobasilar insufficiency in patients with symptoms of cerebral venous dyscirculation. Annals of the Romanian Society for Cell Biology, 315-323. 